\title{
P8: Die Pflegekammer Berlin - eine Konzepterstellung
}

\author{
Marika Lázár
}

Online publiziert: 24. Oktober 2013

(C) Springer-Verlag Wien 2013

Hintergrund: Die Einrichtung einer Pflegekammer zur Stärkung des Pflegeberufs ist mittlerweile in der ganzen Bundesrepublik ein häufig diskutiertes Thema. Zahlreiche Gutachten belegen die Verfassungsmäßigkeit einer solchen Institution. Jedoch stellt sich immer wieder die Frage, ob die in eine öffentlich-rechtliche Selbstverwaltung gesetzten Erwartungen für die Berufsangehörigen der Pflege und für die Öffentlichkeit auch erfüllt werden können. Gibt die Errichtung einer Pflegekammer tatsächlich jedem Berufsangehörigen die Möglichkeit der Mitbestimmung und Mitwirkung? Kann diese dem Allgemeinwohl dienlich sein?

Methode: In dieser Bachelor-Arbeit wurde, unter rechtlichen und betriebswirtschaftlichen Aspekten, ein Konzept über den Aufbau und den möglichen Handlungsfeldern einer zukünftigen Berufskammer in Berlin erstellt. Grundlage boten hier die bestehenden Berufskammern, welche im Berliner Kammergesetz reglementiert sind. Zahlreiche Aufgaben werden im Kammerrecht skizziert und in dieser Arbeit an die, sich in der Pflege ergebenden Kernthemen, angepasst. Weiterhin wurde eine Organisationsstruktur erstellt, welche die Voraussetzung der demokratischen Legitimation erfüllt und somit die ganze Berufsgruppe mit einbezieht. Die Darstellung des finanziellen Aufwandes der Kammer erfolgte in Form eines Kostenplans, um somit Aufschluss über die Höhe der zu erwartenden Mitgliederbeiträge zu erhalten.

Ergebnis und Schlussfolgerung: Da die bestehenden Berufskammern als Erfolgsmodelle angesehen werden, kann davon ausgegangen werden, dass eine Pflegekammer für die Berufsgruppe und die Öffentlichkeit Gleiches leisten könnte. Die demokratische Legitimation ist nur durch die Pflichtmitgliedschaft der Berufsangehörigen zu gewährleisten, gibt jedoch gerade durch diese Pflicht das Recht zur Mitbestimmung und Mitwirkung der Kammertätigkeit. Durch die Motivation vieler Pflegefachkräfte zur ehrenamtlichen Kammerarbeit, ist es möglich, von der vielfältigen Fachexpertise dieser zu profitieren und somit die Qualität der Pflege nachhaltig zu sichern.

M. Lázár $(\triangle)$

Evangelische Hochschule Berlin, Berlin, Deutschland

E-Mail: lazar.marika@yahoo.de 